\title{
2016 Breakthrough Prize and New Horizons in Mathematics Prizes Awarded
}

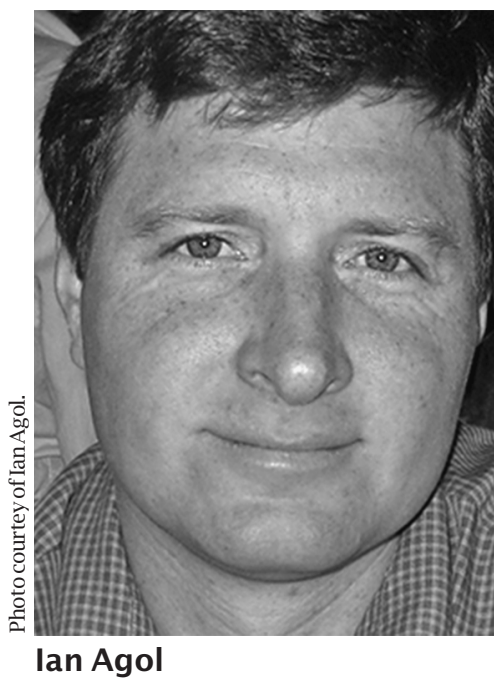

Breakthrough Prize: Ian Agol

IAN AGOL of the University of California Berkeley and the Institute for Advanced Study has been selected as the recipient of the 2016 Breakthrough Prize in Mathematics by the Breakthrough Prize Foundation. Agol was honored "for spectacular contributions to low dimensional topology and geometric group theory, including work on the solutions of the tameness, virtual Haken and virtual fibering conjectures."

The Notices asked David Gabai of Princeton University to comment on the work of Agol. Gabai responded: "Ian Agol is a brilliant mathematician who has made many important and fundamental contributions to such areas as 3-dimensional topology, geometric group theory, hyperbolic geometry, foliation theory, and knot theory. His work utilizes an uncommonly wide range of techniques and methods. Using hyperbolic geometry and 3-manifold topology he proved the Marden Tameness conjecture (independently proved by Danny Calegari and Gabai). That result is crucial to many other results in hyperbolic geometry, such as the ending lamination theorem of Brock-CanaryMinsky. It also proved the Ahlfors measure

For permission to reprint this article, please contact: reprint-permission@ams.org.

DOI: http://dx.doi.org/10.1090/noti1370 conjecture, which had been reduced to Marden's conjecture by Thurston and Canary.

"Agol, with Storm and Thurston, was the first to apply Perelman's work on Ricci flow to a problem outside of geometrization to address many important questions about volumes of hyperbolic 3-manifolds. In particular, their paper gave a new proof of a result of Agol-Dunfield that was used to find the minimal volume closed orientable hyperbolic 3-manifold (Gabai-Meyerhoff-Milley). Agol, with Codá Marques and Neves, used the min-max theory and the Willmore conjecture (Codá Marques-Neves) to solve a long-standing conjecture of Freedman-He-Wang on Möbius energy of links. Using ideas from sutured manifold theory, Agol found an elegant criterion for a 3-manifold to have a finite sheeted cover that fibers over the circle. This, together with the deep work of Dani Wise on quasi-convex virtual hierarchy groups and the foundational work of Haglund-Wise on special cube complexes, proved Thurston's virtual fibering conjecture for Haken hyperbolic 3-manifolds. Agol used geometric group theory (in part with Groves and Manning) and his 3-manifold intuition to solve a conjecture of Wise that implied that all closed hyperbolic 3-manifold groups are quasi-convex virtual hierarchy groups. (This used the Kahn-Markovic solution to the surface subgroup problem and the seminal work of Sageev, as shown by Bergeron and Wise, who proved that fundamental groups of closed hyperbolic 3-manifolds satisfy the hypothesis of Wise's conjecture.) In combination with Agol's virtual fibering criterion, this proved the full Thurston virtual fibering conjecture and hence Waldhausen's virtual Haken conjecture. (That Waldhausen's conjecture reduces to one on hyperbolic 3-manfolds relies on Perelman's geometrization theorem.)

"Beyond solving famous long-standing conjectures, this monumental work proves that the group 
theory of hyperbolic 3-manifolds has tremendous structure not incomparable with that of two-dimensional manifolds. Surfaces have great structure in large part because (except for the sphere) they can be reduced to the disc by sequentially cutting along essential curves. This enables one to prove theorems via induction arguments (often very sophisticated). A consequence of this work is that given any closed hyperbolic 3-manifold, one can first pass to a finite sheeted cover and then cut by a single surface and be left with the product of a surface with the interval. That in turn is the starting point for many deep results in 3-manifold theory."

\section{Biographical Sketch}

Ian Agol was born in 1970 in Hollywood, California. He obtained his PhD in 1998 from the University of California San Diego under the direction of Michael Freedman. He taught at the University of Illinois at Chicago from 2001 to 2007 before joining the faculty at Berkeley. He was awarded the 2009 Clay Research Award (with Danny Calegari and David Gabai). He received a Guggenheim Fellowship in 2005. In 2013 he was awarded, with Daniel Wise, the AMS Veblen Prize in Geometry. He was elected a Fellow of the AMS in 2012.

\section{Response from Ian Agol}

I would like to acknowledge my teachers who encouraged my interest in mathematics. This includes my doctoral advisor, Mike Freedman, whose example encouraged me to be fearless-to work on hard problems and to not be afraid to admit what I do not know. I would also like to acknowledge the many mathematicians whose work mine builds on and to which I've added only a small amount. Their vision encouraged me to go places mathematically that I would not have considered otherwise. I will only single out Daniel Groves and Jason Manning, with whom it has been a pleasure to collaborate. Finally, I'd like to thank my family, especially my wife, Michelle, for her support, and my mother, Maureen, for all the sacrifices she made to get the best education for my brother and me.

\section{About the Prize}

The Breakthrough Prize in Mathematics was created by Mark Zuckerberg and Yuri Milner in 2013. It aims to recognize major advances in the field, to honor the world's best mathematicians, to support their future endeavors, and to communicate the excitement of mathematics to the general public. The prize is accompanied by a cash award of US $\$ 3$ million. As have all five past math laureates, Agol plans to give US $\$ 100,000$ of his prize winnings to support graduate students from developing countries through the Breakout Graduate Fellowships administered by the International Mathematical Union. Previous winners of the Breakthrough Prize are Simon Donaldson, Maxim Kontsevich, Jacob Lurie, Terence Tao, and Richard Taylor (2015).

\section{New Horizons in Mathematics Prize: Larry Guth}

LARRY GUTH of the Mass achusetts Institute of Technology has been awarded a New Horizons in Mathematics Prize for his "ingenious and surprising solutions to

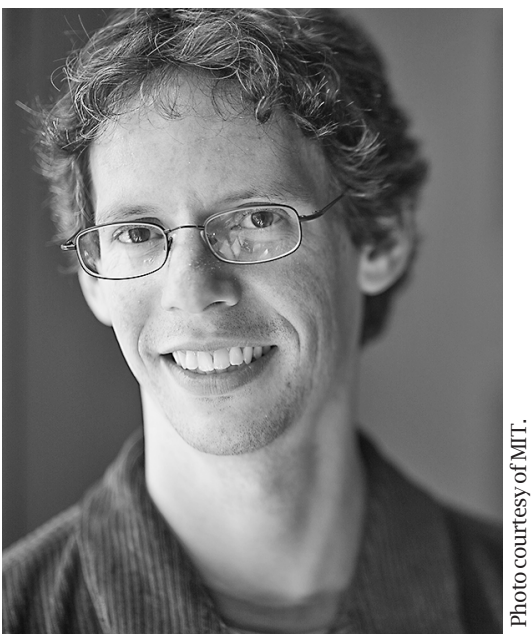
long-standing open problems in symplectic geometry, Riemannian geometry, harmonic analysis, and combinatorial geometry."

The Notices asked Terence Tao of the University of California Los Angeles to comment on the work of Guth. Tao responded: "By introducing powerful techniques from geometry and topology, Guth has solved or made key breakthroughs on many fundamental and difficult problems in harmonic analysis, combinatorics, and (most recently) analytic number theory. For instance, he ingeniously used algebraic topology tools relating to the Ham Sandwich Theorem to prove an important inequality now known as the endpoint multilinear Kakeya inequality, which, roughly speaking, describes the extent to which 'transverse' families of tubes (or tubelike objects) can intersect each other. He has since used this inequality (and variants thereof) for many further applications, most recently in his solution (with Bourgain and Demeter) of the Vinogradov main conjecture on exponential sums, which is connected to such fundamental problems in analytic number theory as Waring's problem (on representing large natural numbers as sums of $k^{\text {th }}$ powers) and bounds on the Riemann zeta function. With Nets Katz, he unexpectedly deployed tools from algebraic geometry (such as the classification of ruled surfaces) to almost fully resolve a famous problem of Erdôs on the minimum number of distinct distances in the plane generated by $n$ points in the plane. Guth's work has given us several new toolboxes of techniques that we will be using to attack further problems in analysis and combinatorics for many years to come." (Note: Tao was among those who nominated Guth for this prize.)

\section{Biographical Sketch}

Larry Guth received his $\mathrm{PhD}$ from the Massachusetts Institute of Technology in 2005 under the direction of Tomasz Mrowka. He held a professorship at the Courant Institute before joining the MIT faculty. He received a Sloan Fellowship in 2010. He was an Invited Speaker at the International 
Congress of Mathematicians in 2010 and a Simons Investigator in 2014. He received the Salem Prize in 2013 and the Clay Research Award (with Nets Katz) in 2015.

\section{Response from Larry Guth}

I want to take this moment to thank my teachers, my collaborators, and my family. I feel very fortunate to have spent time with so many special teachers. I think of them often, especially now that I also teach and I get to try to pass on to my students some of the things that I learned. I've also been very fortunate in my collaborations, and I think that my best work has been collaborative. It has opened up new doors and changed the direction of my career. Finally, I want to thank my family for all their love and support.

\section{New Horizons in Mathematics Prize: André Arroja Neves}

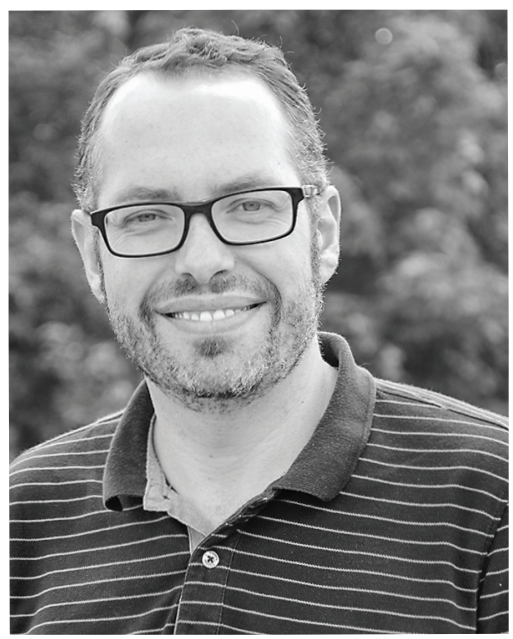

André Arroja Neves
ANDRÉ ARROJA NEVES of Imperial College London has been awarded a New Horizons in Mathematics Prize for his "outstanding contributions to several areas of differential geometry, including work on scalar curvature, geometric flows, and his solution with Codá Marques of the 50-year-old Willmore Conjecture."

The Notices asked Simon Donaldson of the Simons Center for Geometry and Physics at Stony Brook University to comment on the work of Neves. Donaldson responded: "In the ten years from his $\mathrm{PhD}$, André Neves has produced a series of deep results, cutting a wide swath through differential geometry. In one direction, he and Fernando Codá Marques have made spectacular use of variational methods, bringing new ideas and techniques into this venerable field. Fifty years ago, Willmore made a conjecture whose statement can be understood in a first course in surface geometry: the $L^{2}$ norm of the mean curvature of an immersed surface of positive genus in 3-space is at least $\sqrt{2} \pi$. This was a major problem in the subject, attacked by many leading experts and finally solved by Codá Marques and Neves. Their proof is a technical masterpiece of great subtlety. They also used variational methods to establish the existence of infinitely many minimal hypersurfaces in certain Riemannian manifolds, a result to set alongside the renowned classical literature on closed geodesics but which opens up com- pletely new territory. In another direction, Neves is one of the leading experts on Lagrangian mean curvature flow, which is important in connection with Calabi-Yau geometry and mirror symmetry. Among other results, he showed that, in a certain sense, singularities in this flow are unavoidable.

"These are just a few highlights of Neves's work - there are many other important contributions, for example, to the Yamabe functional and to the asymptotic geometry of Riemannian manifolds-and we can be sure that there will be many more to come. His work is characterized by exceptional technical power, combining ingenious geometrical constructions with deep results from analysis and PDE."

\section{Biographical Sketch}

André Neves was born in 1975 in Lisbon, Portugal, and received his $\mathrm{PhD}$ from Stanford University in 2005 under Richard Schoen. He is a recipient of the Leverhulme Prize (2012), the Whitehead Prize of the London Mathematical Society (2013), and the AMS Veblen Prize (with Fernando Codá Marques) in 2016. He was an Invited Speaker at the International Congress of Mathematicians in 2014.

\section{Response from André Arroja Neves}

Twenty years ago I was very fortunate to find mathematics and fall in love with it. It has been a great journey so far, with its adequate share of surprises and disappointments, new paths and dead ends. I haven't traveled it alone, and two mathematicians have had a great influence on my career: my advisor, Rick Schoen, a constant source of inspiration, and my collaborator, Fernando Marques, with whom discovering mathematics together has been a tremendous pleasure. I must also thank Filipa, as none of this would have any meaning without her tremendous generosity and unyielding support, and our two little children, Eva and Tomás, who effortlessly fill my days with constant joy.

\section{About the Prize}

The New Horizons in Mathematics Prizes were created in 2015 to recognize promising junior researchers who have produced important work. Up to three of these prizes will be given each year. These prizes carry a cash award of US\$100,000, each.

-Elaine Kehoe 